\title{
Swarming Models for Facilitating Collaborative Decisions
}

\author{
C.B. Zamfirescu, F. G. Filip
}

\author{
Constantin-Bala Zamfirescu \\ Lucian Blaga University of Sibiu, \\ Faculty of Engineering, Department of Computer Science and Automatic Control \\ Romania, 69121 Sibiu, 17 Emil Cioran \\ E-mail: zbc@acm.org \\ Florin Gheorghe Filip \\ 1. Romanian Academy - INCE and BAR \\ Romania, 010071 Bucharest, 125 Calea Victoriei, and \\ 2. Nat. Institute for Informatics -ICI \\ Romania, 011455 Bucharest, 8-10 B-dul Maresal Al. Averescu \\ E-mail: filipf@acad.ro
}

\begin{abstract}
:
The paper highlights the computational power of swarming models (i.e., stigmergic mechanisms) to build collaborative support systems for complex cognitive tasks such as facilitation of group decision processes (GDP) in e-meetings. Unlike traditional approaches that minimize the cognitive complexity by incorporating the facilitation knowledge into the system, stigmergic coordination mechanisms minimize the complexity by providing the system with emergent functionalities that are shaped by the environment itself through the possibility to structure it in terms of high-level cognitive artefacts. This is illustrated by conducting a socio-simulation experiment for an envisioned collaborative software tool that acts as a stigmergic environment for modelling the GDP. The results show superior results when the users are allowed to increase the representational complexity of a GDP model with cognitive artefacts that support guidance and action in the conceptual problem space.
\end{abstract}

Keywords: collaborative working environments, group decision support systems, facilitation, social simulation, stigmergy, swarming models of computation.

\section{Introduction}

The simplicity of swarming models is becoming ever more popular in the design of decentralized systems that are developed to run in open, dynamic, and unknown environments. Inspired from the behaviour of social insects, marine animals, birds, and even humans, the concept covers a broad spectrum of mechanisms able to generate an intelligent collective behaviour. These mechanisms are simply identified as stigmergic coordination[1].

The most cited example of swarming models is the food foraging behaviour in ant colonies [2]. Each ant senses the signs (pheromones) in its environment and acts in accordance with them without any direct communication with other ants from the colony. If there is no sign in the environment the ant executes a randomized search for food. When an ant discovers a food source, it drops a smelling chemical substance (pheromone) on its way back to the nest while carrying a bit of food. Thus the ant creates a pheromone trail between nest and food source. When an ant senses the pheromone trails, it will follow the most intense one to the food source, the intensity of the pheromone signifies the shortest path toward the food that was discovered until that point in time; when the ant arrives at the food source, it will return with food, while depositing more pheromones, intensifying the pheromone trail. The above 
simple reactive behaviour of each ant results in an emergent intelligent behaviour of the colony that is able to find the shortest path from the nest to the food source without any central coordination. This behaviour is constrained by the limited sensorial aptitude of an ant to sense the local pheromone trails with no mental plan on how to find the shortest path or knowledge about the environment in which they act. Consequently, the ant's behaviour is an emergent property induced at the same time by two exogenous factors: 1) the environment (the surface of the terrain and the evaporation mechanism for the pheromones), and 2) the ants' actions over the environment (the pheromone trails).

Despite its behavioural simplicity (i.e., stimuli-response rules), a single stigmergic agent can emulate any Turing machine and can execute any symbolic or sub-symbolic algorithm proposed by the AI (artificial intelligence) research mainstream [3]. Nevertheless, most of the computer applications are outside the domain of human cognitive abilities, serving merely as cognitive support systems through an active and semantically rich environment [4]. Therefore swarming models of computation are seen as a feasible approach to construct systems that are not limited to the classical optimization problem of finding the shortest route [5], but are able to support human decisions as well $[6,7,8]$.

One application domain with a high level of cognitive complexity is the facilitation of GDP in emeetings. The complexity associated with the construction, coordination and execution of GDP is well recognized in the research field of Group Decision Support System (GDSS) [9]. For this reason GDSS has seldom been a full success story and a widely adopted technology as it has been foreseen by its pioneers. All the applications developed to support the group facilitation in e-meetings follow the traditional centralized approach where the system explicitly codifies the facilitation knowledge. Examples include software tools that embed knowledge about the collaborative patterns of interaction [10] and workflows [11] for the most frequently used GDPs. These applications basically suffer from the same obstacles met in the traditional AI mainstream such as [12]: 1) the restrictions to codify the human's knowledge into the computing system; 2) the lack of self-development capabilities for this knowledge; 3 ) the blackbox perspective over a system disconnected from the environment where the relevant knowledge are extracted.

In contrast to the traditional approach that codifies the facilitation knowledge into the GDSS, this paper illustrates how the stigmergic mechanisms of swarming models may be employed to build emergent and self-organizing functionalities that support group facilitation in e-meetings. In this particular case, the stigmergic coordination mechanisms are implemented over the conceptual environment of the GDP modelling space which is exploited by the users of an e-meeting system. Unlike conventional approaches that minimize the cognitive complexity associated with the construction and execution of a GDP by incorporating the facilitation knowledge into the system, stigmergic coordination mechanisms minimize it by structuring a shared conceptual environment populated with the cognitive artefacts that represents the basic skills of conducting an e-meeting. In this way the users are collectively constructing and interpreting the facilitation knowledge through the successive uses of the system.

The remaining part of this paper is organized as follows. The next section presents a brief analysis of the innate relationship between distributed cognition and stigmergy as it has been presented by many authors within the Web 2.0 technology mainstream. Section 3 describes the main components of an envisioned collaborative software tool that act as a stigmergic environment for modelling the GDP: the structure of the semantic environment, the low-level behaviour of the users in interacting with this environment, and the high-level of cognitive constructs that may be employed by the users to structure the GDP. These components are implemented and tested in a socio-simulation experiment which is described in Section 4. The experimental results show clear self-organizing capabilities, but simultaneously high dependability of system's performance on the user's ability to structure the stigmergic environment. From the engineering standpoint of constructing purposeful facilitation tools for e-meetings, these results are discussed and summarized in the last section. 


\section{Stigmergy and cognition}

Most of the research in cognitive science is rooted in the basic assumption that cognition is purely an internal process of representation and manipulation of knowledge disconnected from the environment. Nevertheless, recent studies in cognitive sciences reveal the essential role of the environment (physical or artificial) in mediating the knowledge by facilitating their external representation and information exchange among these representations [13]. It becomes clear 'that individuals are socially and culturally situated and that the environment needs to be considered in order to understand cognition' [14].

By admitting the critical role of the environment in cognition, the researchers became increasingly aware of the relationship between cognition and stigmergy. Even if the term stigmergy has been primarily used for typically reactive (non-rational) agents, its relationship with cognition was investigated for the first time by Susi and Ziemke [15]. The authors conclude with the assertion that the conceptual framework of stigmergy offers a common denominator for the social sciences theories (i.e., activity theory; situated and distributed cognition). For example a cave painting is an emblematic case of stigmergic coordination where people used the physical environment to indirectly communicate their knowledge in ancient human society. The relevance to cognition of the stigmergic coordination mechanisms in human society is illustrated by Parunak in several examples of social activities [6].

Albeit the use of stigmergic mechanisms may be observed in various social activities, it is more evident in the digital world realm. In a comprehensive study of collaborative support systems, Elliot [16] found that stigmergy is a coordination mechanism inherent not only in collaborative processes over physical environments, but also in a range of collaborative support systems. For instance the plethora of applications that are considered to be Web 2.0 technology (especially media such as wiki and community blogging) is generally recognized to be stigmergic systems. Moreover, applications such as Google's PageRank system, eBay's online auctioning, Amazon's recommender systems [6], Wikipedia.org, open source software and multiplayer social environment Second Life [17] are employing the stigmergic coordination mechanisms to exhibit the functionalities of an intelligent collective behaviour.

In these stigmergic systems the users exploit their digital environment through the use of engineered artefacts that may be annotated with symbolic information representing the human's cognition [7]. Given that the essential capability of any stigmergic system is to transfer the cognitive complexity from the humans to the environment [18], the problem-solving capabilities of the users decisively depend on how the problem is represented in the digital environment. A standard representation of the problem in the environment is realized as a composition of cognitive artefacts linked in a weighted graph. Basically, this graph signifies a navigation map that supports the cognitive effort to find and reach any artefact from the place where it is needed. The artefacts commonly stand for the possible states of the problem, while the links are the set of possible actions that guide the decision process (the conceptual navigation) from one state to another of the problem space. Similar with the intensity of the pheromone trails in the case of real ants, these actions are weighted in order to discriminate the most effective ones. As a result, the improvement of problem-solving ability requires two corresponding processes [8]: 1) the augmentation of the environment with additional states and actions to increase the accuracy of problem representation, and 2) the improvement of the preference function for an action in order to compensate the expansion of the exploration space.

The wide employment of the stigmergic patterns of interaction in collaborative working environments has been triggered by its fundamental advantage of preventing the humans' cognition to be exposed to the complexity of the environment [19]. As users interact only locally, there is no need for tasks allocation, the tasks being preferentially performed by the most expert since they are the most attracted to act and finalize the task with minimal effort [20]. Moreover there is no need for prediction since the environment records actions in the problem space and the unexpected events are automatically traced through the outcome of the users' actions over the environment. All these advantages make the stigmergic models of computation a suitable approach to support the cognitive complexity of facilitating GDP, a process that 
runs in a dynamic, open and uncertain environment.

\section{A simulation model for group decisions in e-meetings}

The wide range of tools that support group decisions in e-meetings falls under the GDSS general umbrella term. GDSS is defined as an interactive computer-based environment that supports a concerted and coordinated team effort towards completion of joint tasks [21]. A GDSS is composed of a set of highly configurable collaborative "tools" (e.g., brainstorming, voting and ranking, multi-criteria analysis, etc.) that requires a high level of expertise for an effective use for complex decisions [22]. The strong relationship between the GDP outcome and the presence of a skilful facilitator to direct the joint decision process is thoroughly presented in many field studies of GDSS research [23]. The inaccessibility of many organizations to a well-trained GDSS facilitator is recognized to be one of the main obstacles which limit the adoption of GDSS technology [24]. To reduce the dependence on the facilitator, the participant-driven GDSS was proposed as the most promising research direction to leverage the skills and abilities of each group member [25]. However, this approach is highly constrained by the cognitive complexity associated with the construction, coordination and execution of GDP by inexperienced users.

To overcome the problem of cognitive complexity Briggs and de Vreede [24] introduced the thinkLet (TL) concept as a discrete facilitation unit that integrates a specific tool, its configuration and a script to use it - a predefined interaction protocol among users that is enforced and mediated by a specific collaborative tool. This concept was anticipated by the declarative model of the experienced decision maker (MEDM), which was proposed in late 80's to help the user of a DSS to build the model, select the appropriate solver, and evaluate various solutions provided by the computerized algorithms in so called "mixed knowledge" DSS [22, 26]. The TLs are considered to be the smallest piece of essential knowledge to design collaborative processes. Examples include [24]: StrawPoll (Evaluate) - used to reveal the agreements or disagreements within a group; Leafhopper (Diverge) - used when the participants does not known in advance the topics for discussion or they have different interests or level of expertise; ExpertChoice (Organize) - used when the ideas of the group are organized by a single participant; ReviewReflect (Converge) - used when a different reviews from group members for a document should reach an agreement etc. With TLs, the conceptual model for a GDP takes the form of a shared plan of collaborative actions [24, 27]. Each collaborative action is an interaction protocol embodied in a TL. As any plan, the model for a GDP may be hierarchically decomposed in sub-plans at different levels of abstraction. This conceptual structure of a GDP model is acknowledged in any application domain of the GDSS technology, such as project management [28], user requirement elicitation [29], crisis response management [30], scenario design [31], risk identification [32], etc.

In the view of envisioning a collaborative software tool that acts as a stigmergic environment for modelling the GDP (in the same way in which a collaborative CAD software acts as a stigmergic environment for architectural design [33]), we developed a socio-simulation model that mimics the users' conceptual 'navigation' over the semantic structure of the problem space for facilitating the e-meetings. As for any stigmergic system the simulation model entails the description of agents' behaviour and the structure of the shared environment where the agents are localized and moved over it.

For the GDSS domain, the agents are the users responsible to define, execute and evaluate a GDP model which is a path through the conceptual space of the available TLs. The environment is the collaborative facilitation tool that supports the conceptual representation of the problem space comprising all the TLs discovered and documented by the users' community (so far there are over 70 TLs acknowledged in literature [34]). 


\subsection{The semantic environment for facilitating the e-meetings}

According to Parunak [6], a stigmergic environment assumes the definition of three main components: 1) topology, 2) states, and 3) processes. Structurally, the topology may be viewed as a fully connected weighted graph that codifies the facilitation knowledge of group decision in e-meetings. This knowledge presumes correlated information among the users and the TLs, reflecting the users' evaluation of the performance for a TL (a node in the graph) relative to a problem type. The performance is stored for each problem type in a variable associated with each edge of the graph. The problem type is simply codified through a unique $I D$ to distinguish among different performances when they are read, during the modelling phase of the GDP, or modified, after the GDP has been executed and evaluated by agents. Evaluation of a GDP model entails a subjective assessment of the model against some performance criteria after its execution. Regularly, GDP's performance may be quantified in terms of efficiency, effectiveness and users' satisfaction, as illustrated in several studies from the GDSS research field [35].

The performance from all the graph's edges describes the state of the environment over time. Usually, the environment executes a set of processes on the variables (as aggregation end evaporation in the case of ants). For our case, we apply a simple weighted additive rule to simulate the aggregation of performances:

$$
P_{j k}\left(T L_{k}, t\right)=P_{j k}\left(T L_{k}, t-1\right)+U P_{j k}\left(T L_{k}\right) / w
$$

where: $t$ represents the temporal component of the model which is incremented by one for each successive use of the GDSS; $k$ is the TL's identification index from the set of TLs used to model the $\mathrm{GDP} ; U P_{j k}\left(T L_{k}\right)$ - is the user's performance of the $k$-th TL evaluated from the side of TL $j$ at moment $t ; P_{j k}\left(T L_{k}, t\right)$ and $P_{j k}\left(T L_{k}, t-1\right)$ are the new and previous values of the (collective) performance stored on the edge between the TLs $j$ and $k$; $w$ is a tuning parameter, arbitrarily chosen, to weight the impact of the last evaluation.

\subsection{The agents' behaviour over the semantic environment}

The agents are the users who interact with the envisioned collaborative tool to model the GDP. Conceptually, in any point in time an agent is "located" in a node (TL) of the cognitive environment of the problem space, performing one of the following basic actions: 1) evaluates the preference for the next possible TL (or TLs) that are going to be executed given the current execution context of the GDP; 2) selects the next best TL (or a group of TLs) for further completing the GDP model; 3) executes the TL (or the group of TLs) from the model, and finally; 4) evaluates the performance for the executed TLs. The evaluation activity is simulated using the formula (1), while the first three actions with Luce's selection axiom [36]:

$$
p_{j k}=\mathrm{e}^{p_{j k}\left(T L_{k}\right) / T} / \sum_{i=1}^{m} \mathrm{e}^{p_{j i}\left(T L_{i}\right) / T},
$$

where $p_{j k}$ represents the preference for an alternative TL, i.e. the selection probability of the TL $k$ from the TL $j ; i$ is the index of TLs connected from the side of node $j$ (in fact all the $m$ TLs available in the problem space as long the graph is fully connected); and $T$ is a parameter used to define the deviation from a pure rational behaviour.

The above formula is the most common model of stochastic decisions due to its correlation with the psycho-social observations of human behaviour in several domains. As a result of normalization, the preferences for the unexploited TLs are diminishing after each performance update. This mechanism replicates the pheromone evaporation process of the real ants (e.g., even if a TL has been positively evaluated after an execution of a GDP model, the associated preference will decrease once a better alternative is discovered and more frequently used). The uncertainty associated with the construction of preferences is generally modelled in equation (2) with the parameter $T$ that range between 0 (when 
selection is deterministic as is the ideal case of a perfectly informed decision) and 1 (when the selection is completely random as in the case of a completely irrational decision). Note that Luce's selection axiom does not specify the reasons of uncertainty which for the modelling of GDP may cover any aspect of complexity, unfeasibility, cost or even refusal to evaluate the performance of a TL after its execution.

\subsection{Navigation strategies over the semantic environment}

The agents, who are reflecting the users in modelling a GDP, are engaging in means-ends reasoning activities to achieve the group decision goal. During the execution of the model they must be able to adapt to the changes and uncertainties associated with both the execution and the decision goal. Moreover, when the decision problem is not completely clear or too complex to be fully tackled, the users are defining intermediate sub-goals to be subsequently achieved. As a result, the design of a GDP model is most often done incrementally and interleaved with its execution.

Table 1: Different modelling strategy to construct a GDP model

\begin{tabular}{l|l|l|l}
\hline \hline $\begin{array}{l}\text { Design strategies } \\
\text { (DS) }\end{array}$ & Execution & Problem's goal & Codification \\
\hline \hline DS1 & Certain & Stable & $\begin{array}{l}\text { The problem type is codified through } \\
\text { a unique ID }\end{array}$ \\
\hline DS2 & Uncertain & Stable & $\begin{array}{l}\text { The problem type is codified as a } \\
\text { variation from the current state of } \\
\text { execution to the desired one }\end{array}$ \\
\hline DS3 & Uncertain & Unstable & $\begin{array}{l}\text { The problem type is codified as a } \\
\text { variation from the current state of } \\
\text { execution to any future possible state }\end{array}$ \\
\hline \hline
\end{tabular}

In Table 1 we have summarized three basic design strategies (DS), with direct implications on the way the GDP model is decomposed on different levels of abstractions. These are:

- DS1 which corresponds to the traditional use of GDSS when the GDP model is predefined by the facilitator. In this case, the user is providing a complete structure of the GDP model, the facilitator having a complete vision over the execution plan for the GDP. It includes all the necessary collaborative actions (in the form of TLs) together with their precedence constraints. This design strategy relates to the hypothetical conditions when the execution context remains stable in time as regards the TL execution's outcome and the decision's objectives. Thus, each problem type is identically codified in all edges that connect the TLs used in modelling the GDP and relates to a low level of semantically structured environment.

- DS2 which corresponds to problem types with stable objectives but uncertain TL execution's outcome from the GDP model. In this case, after the execution of each TL, adjusting the remaining GDP is needed. This design strategy is codified in the conceptual graph of GDP modelling with different $I D s$ for each sub-problem that corresponds to the variance from the current state of execution to the desired one.

- DS3 which relates to complex plans of actions for the GDP model, where both the outcome of an activity and the decision's objectives are unstable during the execution. In this case, when the decision problem is not entirely clear or too complex for designing an entire GDP model, the users are defining intermediate sub-goals that are subsequently dealt with. This design strategy is codified in the conceptual graph for the GDP modelling with different $I D s$ for each sub-problem that corresponds to the variance from the current state of execution to any future possible one. 


\section{Experimental results}

To evaluate the design strategies for modelling the GDP we conducted a virtual experiment following the research methodology proposed by Carley [37], implementing in the Netlogo multi-agent simulation environment [38] the model described in the previous section. In the experiment the users ("turtles") are to engage themselves in the facilitation of e-meetings, trying to define the GDP model for a problem type by moving in the conceptual graph of TLs (the nodes and edges are implemented also as "turtles"). The number of TLs that compose the graph is arbitrary chosen from the interface (the "num-TLs" variable in Figure 1), while their utilities for a certain problem type is predefined with random values between 0 and 1 when the experiments are initialized. Note that the NetLogo implementation includes some additional variables required to analyse if the model presents similar results to those reported in the traditional ethnographical studies. This issue is beyond the scope of this paper and is detailed in [39].

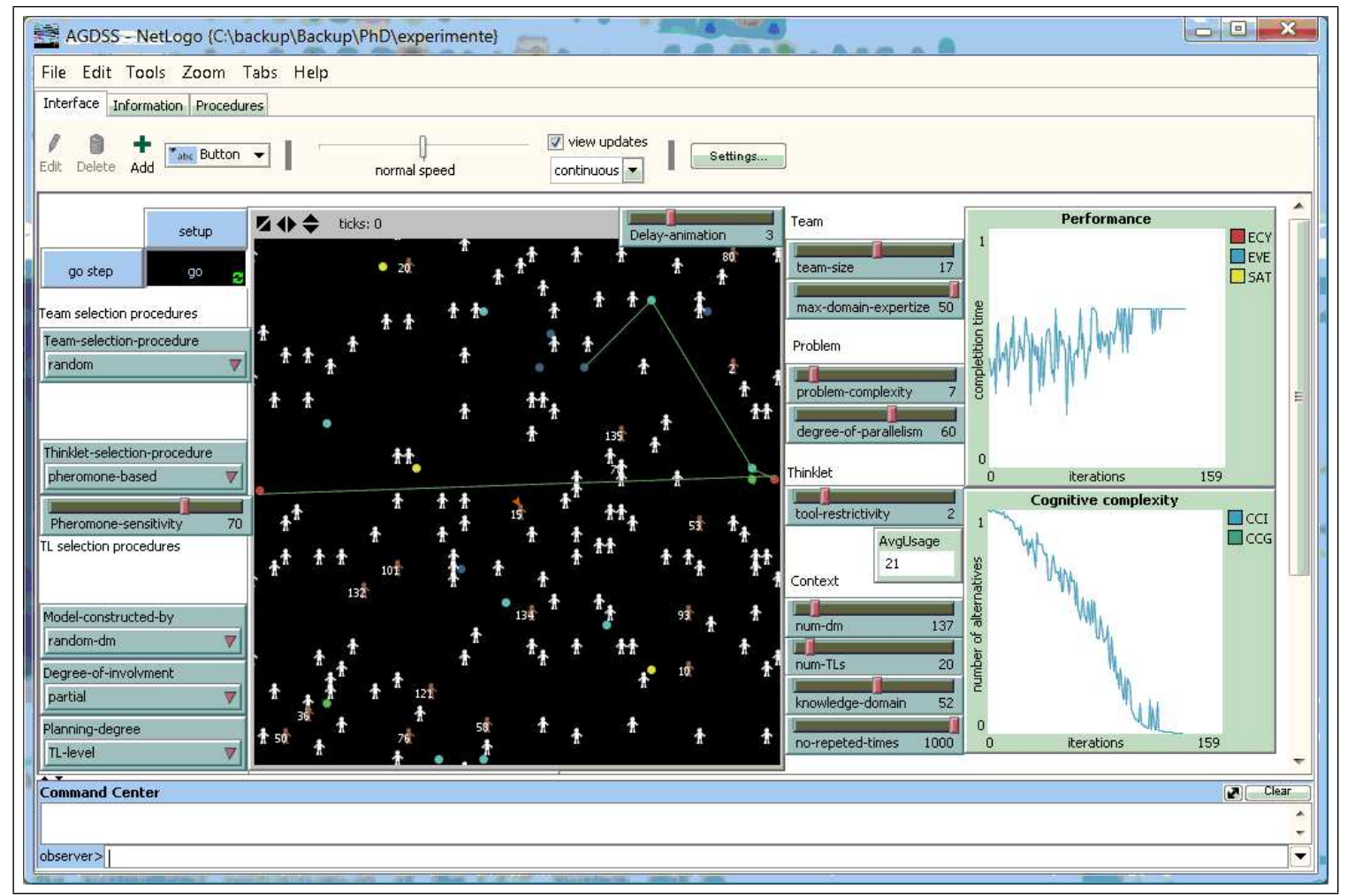

Figure 1: The interface of the model in the Netlogo environment

In the next sections the normalized performance of the GDP models and its associated entropy for 100 successive explorations (iterations) in the three design strategies (the "Planning-degree" variable in Figure 1): $D S 1, D S 2$ and $D S 3$ are presented. An exploration stands for a complete execution cycle of a GDP. It includes three consecutive phases: 1) finding a suitable model through the successive selection (using the Equation (2)) of TLs that compose the GDP for the given problem type; 2) executing the identified model and assessing its performance by reading and averaging the predefined utility values of all the TLs that compose the GDP model; 3) evaluating the model by updating the performance value (using the Equation (1)). The statistics are aggregated from 30 experiments for a relatively simple problem type of 5 successive TLs. The parameter $T$ from Equation (2) is set to 0.7 (the "pheromonesensitivity" variable from Figure 1 to favour a faster convergence rate in finding a suitable solution in the problem space composed of 70 TLs.

From the engineering viewpoint, the design strategies have direct implications on the way in which the cognitive environment for modelling the GDP is structured. As described before, the structure of 
the environment is simply reflected in the possibility to semantically decompose the problem in subproblems on different levels of abstractions. This design issue implies in our implementation to record with specific $I D s$ the performance for each sub-problem type that emerges from the decomposition process.

\subsection{The impact of the modelling strategies over the model's performance}

In Figure 2 the aggregate performance (a relative number between 0 and 1 as resulted from averaging the predefined utility values of all the TLs that compose the GDP model) from 30 experiments of a GDP modelling process for the defined design strategies DS1, DS2 and DS3 are shown. As may be expected, the performance fits an exponential function, a typical behaviour for a stigmergic system [40, 41, 42].

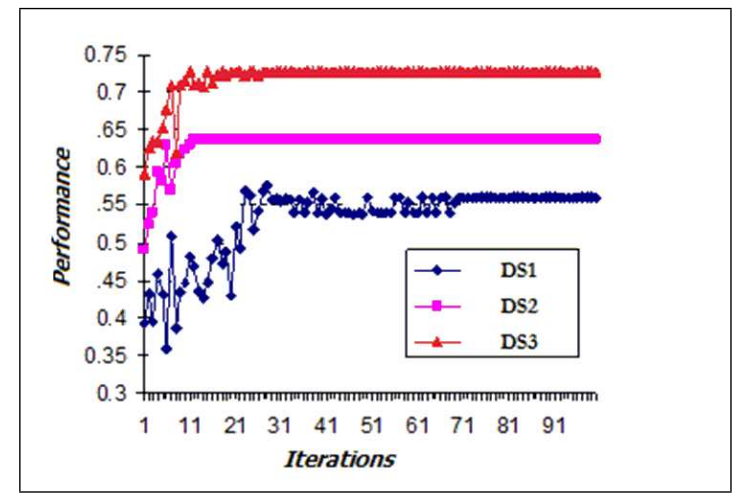

Figure 2: The GDP models' performance for the defined modelling strategies

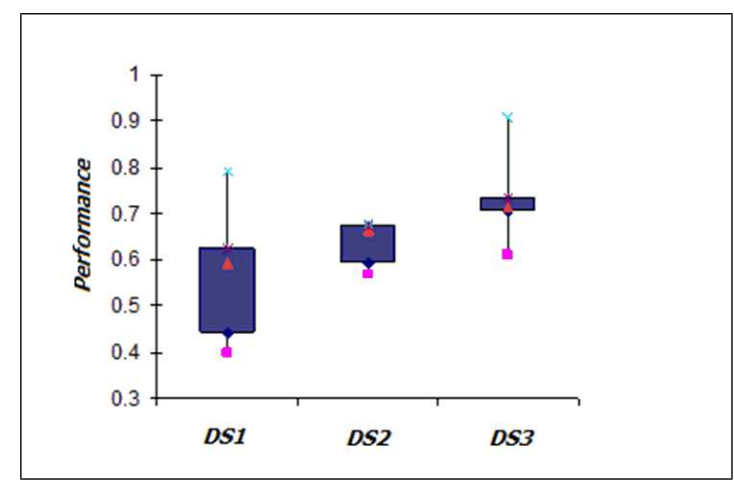

Figure 3: The distribution of GDP models' performance for the three modelling strategies

Like any heuristic model, the stigmergic coordination mechanisms do not guarantee finding an optimal solution, but a near-optimal or acceptable one. As a consequence, from one experiment to another there are some variations in performance for the convergence values. Figure 3 illustrates in Whiskers diagrams the distribution of performance for the same experimental data that are depicted in Figure 2.

The three strategies show different performance and convergences to an optimal solution. Contrasting with $D S 1, D S 2$ takes benefit from the prior experiences not only in relation with the entire problem type but also from the intermediate solutions to model the sub-problems in which the initial problem has been decomposed. In addition, $D S 3$ increases the granularity by adding the opportunity to decompose the problem's objective. As a consequence, the figures show the influence of problem type decomposition on the GDP model's performance.

As may have been expected, $D S 3$ shows the best performance results and a low inconsistency among the identified solution for a GDP model. But, DS2 converge faster to an optimal solution and a lower 
inconsistency among the feasible solutions (the deviation of the average solution from those with maximal and minimal performances). This can be explained by the additional constraints of having stable objectives during the modelling process.

\subsection{The cognitive complexity associated with each modelling strategy}

The auto-organization of relations between TLs (i.e., the performance update after successive evaluations) entails a decrease of freedom due to the emergence of contextual constraints that reduce the probability to select some TLs (i.e., the preference for the available TL as defined in Equation (2)). For a problem type, the degree of freedom corresponds to the probabilistic distribution of preferences for the selection alternatives that is equivalent with the Shannon normalized entropy [40, 43]. The Shannon normalized entropy for the selection of a TL is given by:

$$
E\left(p_{\mathrm{jk}}\right)=-\sum_{k=1}^{m} p_{\mathrm{jk}} \cdot \ln \left(p_{\mathrm{jk}}\right) / \ln (m)
$$

where $p_{\mathrm{jk}}$ - represents the preference, the selection probability of the TL $k$ from the TL $j ; k$ - is the index for the TLs connected from the node $j$ (in fact, all the $m$ TLs available in the problem space).

When the recorded performance is equal for all the available modelling alternatives, the user is considering the entire problem space when he selects a feasible TL (the probabilities from Equation (3) being equally distributed entail an entropy equal with 1). Contrary, when the recorded performance favours a single alternative, the user will have no freedom in the selection of the best TL (all the probabilities from formula 2 being 0 except the best alternative that is 1 , entails an entropy equal with 0 ). Thus, the entropy associated with TL's selection is a measure of cognitive complexity for modelling the GDP. Moreover, it is a local metrics that can be computed for each TL's selection activity for modelling the GDP.

Figure 4 shows the cognitive complexity associated with GDP modelling for the design strategies: $D S 1, D S 2$ and DS3. The data are obtained for the same experimental settings as introduced in the beginning of this section. Because this measure is computed on the basis of the local data for each selection action (the performances available on the edges from the current TL), the figure corresponds to the average of entropies for all the TL selection actions needed to complete the GDP model (5 successive TLs, for this case).

Similar to the distribution of GDP models' performance, comparing with DS1 the normalized entropy for $D S 2$ and $D S 3$ converge faster to 0 . For $D S 3$ the gradual increase in the complexity of the representations for a GDP model in the conceptual problem space (through the definition of different sub-plans on different semantic levels) makes the design of the GDP process more manageable in the sense that it reduces the overall complexity of representing the GDP model. Nevertheless to reduce the cognitive complexity for modelling the GDP, the users should be supported in using this representation for guidance and action in the conceptual problem space. This entails the reflection of the abstract representations into the conceptual environment through an increased semantic complexity (by adding new problem types, codified with distinct IDs in our implementation) which facilitates the synergy among partially overlapping GDP. Only in this way the users may move from one sub-plan to another or from low level detailing with TLs to high level of plan and to be fully supported by the stigmergic coordination mechanisms at the same time.

\section{Summary and conclusions}

Stigmergic mechanisms are widely employed in human society, from the classical examples of largescale complex adaptive systems such as stock markets, supply logistics and cultural memes to the more 


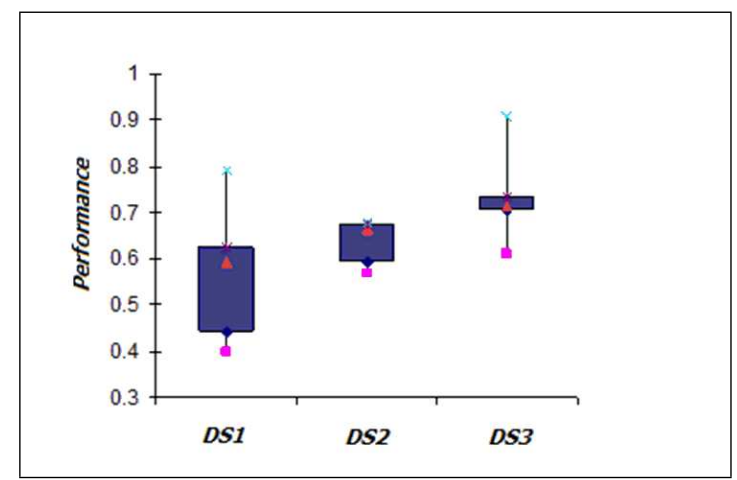

Figure 4: The normalized entropy of the GDP modelling for the three design strategies

recent range of collaborative working environments such as wiki, Google, social networking and opensource software.

Continuing our work in achieving inter-paradigmatic synergy between symbolic and sub-symbolic reasoning for large-scale complex systems [44, 45], the paper highlighted the computational power of stigmergic coordination mechanisms to build collaborative support systems for complex decisions such as facilitation of GDP in e-meetings. Unalike conventional approaches that minimize the cognitive complexity by incorporating the facilitation knowledge into the system, stigmergic coordination mechanisms minimize it by offering emergent functionalities that are made up not only by the user's actions (through the selection of the right modelling components) but also by the environment itself (through the possibility to structure it in terms of high-level cognitive artefacts such as plans or sub-plans). This was demonstrated by implementing and testing in a socio-simulation experiment an envisioned collaborative software tool that act as a stigmergic environment for modelling the GDP. The results show superior results when the users are supported to gradually increase the complexity of the representations for a GDP model which is reflect on its turn in the relational complexity of the conceptual problem space.

\section{Bibliography}

[1] P.P. Grassé. La theorie de la stigmergie: essai d'interpretation du comportement des termites constructeurs, Insectes Sociaux, 6: 41-81, 1953.

[2] P. Valckenaers, H. Van Brussel, Hadeli, O. Bochmann, B. Saint Germain, C. Zamfirescu On the design of emergent systems: an investigation of integration and interoperability issues. Engineering Applications of Artificial Intelligence, 16(4): 377-393, 2003.

[3] H.V.D. Parunak, S.A. Brueckner. The Cognitive Aptitude of Swarming Agents http://www.newvectors.net/staff/parunakv/CASA.pdf, 2009.

[4] M.A. Rodriguez. Faith in the Algorithm, Part 1: Beyond the Turing Test, http://faithinthealgorithm.net/, 2009.

[5] S.C. Negulescu, C.V. Kifor, C. Oprean. Ant Colony Solving Multiple Constraints Problem: Vehicle Route Allocation, International Journal of Computers, Communications and Control, 3(4): 366-373, 2008.

[6] H.V.D. Parunak. A Survey of Environments and Mechanisms for Human-Human Stigmergy, Lecture Notes on Artificial Intelligence, 3830: 163-186, Springer, 2006. 
[7] A. Ricci, A. Omicini, M. Viroli, L. Gardelli, E. Oliva. Cognitive Stigmergy: Towards a Framework Based on Agents and Artifacts, Lecture Notes in Computer Science, 4389: 124-140, Springer, 2007.

[8] F. Heylighen, J. Bollen. The World-Wide Web as a Super-Brain: from metaphor to model, Proceedings of Cybernetics and Systems '96, Austrian Society for Cybernetics, 917-922, 1996.

[9] G.J. de Vreede, J.A. Boonstra, F. Niederman. What Is Effective GSS Facilitation? A Qualitative Inquiry Into Participants' Perceptions, Proceedings of the $35^{\text {th }}$ Hawaiian Internal Conference on System Sciences, IEEE Computer Society Press, 2002.

[10] G.L. Kolfschoten, W. Veen. Tool Support for GSS Session Design, Proceedings of the $38^{\text {th }}$ Annual Hawaii International Conference on System Sciences, IEEE Computer Society Press, 2005.

[11] A.V. Lopez, Q. Booker, N.S. Shkarayeva, R.O. Briggs, J.F. Nunamaker Jr.. Embedding Facilitation in Group Support Systems to Manage Distributed Group Behavior, Proceedings of the $35^{\text {th }}$ Hawaii International Conference on System Sciences, IEEE Computer Society Press, 2002.

[12] R.A. Brooks. Intelligence Without Representation, Artificial Intelligence Journal, 47: 139-159, 1991.

[13] E. Hutchins. Cognition in the wild. Cambridge, MIT Press, 1995.

[14] J. Rambusch, T. Susi, T. Ziemke. Artefacts as Mediators of Distributed Social Cognition: A case study. Proceedings of the $26^{\text {th }}$ Annual Conference of the Cognitive Science Society, 1113-1118, Mahwah, NJ: Erlbaum, 2004.

[15] T. Susi, T. Ziemke. Social Cognition, Artefacts, and Stigmergy: A Comparative Analysis of Theoretical Frameworks for the Understanding of Artefact-mediated Collaborative Activitym, Cognitive Systems Research, 2(4): 273-290, 2001.

[16] M.A. Elliott. Stigmergic Collaboration: A Theoretical Framework for Mass Collaboration, PhD Thesis, Centre for Ideas, Victorian College of the Arts, The University of Melbourne, 2007.

[17] M.A. Elliott. Stigmergic Collaboration: The Evolution of Group Work,M/C Journal, 9(2), http://journal.media-culture.org.au/0605/03-elliott.php, 2006.

[18] H.V.D. Parunak, T.C. Belding, R. Bisson, S.A. Brueckner, E. Downs, R. Hilscher. Stigmergic reasoning over hierarchical task networks. Proceedings of The $8^{\text {th }}$ International Conference on Autonomous Agents and Multiagent Systems, 1195-1196, 2009.

[19] I. Dzitac, B.E. Barbat, Artificial Intelligence + Distributed Systems = Agents, International Journal of Computers, Communications and Control, 4(1):17-26, 2009.

[20] F. Heylighen. Accelerating Socio-Technological Evolution: from ephemeralization and stigmergy to the global brain. in: Globalization as an Evolutionary Process: Modeling Global Change, edited by George Modelski, Tessaleno Devezas, and William Thompson, London: Routledge, 286-335, 2007.

[21] G. DeSanctis, B. Gallupe. A Foundation for the study of Group Decision Support Systems, Management Science, 589-609, 1987.

[22] F.G. Filip, Decision support and control for large-scale complex systems, Annual Reviews in Control, 32(1): 61-70, 2008. 
[23] F. Niederman, C.M. Beise, P.M. Beranek. Issues and Concerns about Computer-Supported Meetings: The Facilitator's Perspective, MIS Quarterly, 20(1): 1-22, 1996.

[24] R.O. Briggs, G.J. de Vreede, J.F. Nunamaker, Jr.. Collaboration Engineering with ThinkLets to Pursue Sustained Success with Group Support Systems, Journal of Management Information Systems, 19(4): 31-63, 2003.

[25] J.H. Helquist, J. Kruse, M. Adkins. Developing large scale participant-driven group support systems: An approach to facilitating large groups, Proceedings of the First HICSS Symposium on Field and Case Studies of Collaboration, IEEE Computer Society Press, Los Alamitos, CA, 2006.

[26] F.G. Filip. System analysis and expert systems techniques for operative decision making. J. of Syst. Anal. Model. Simul. 8(2): 296-404, 1990.

[27] C.B. Zamfirescu. An Agent-Oriented Approach for Supporting Self-Facilitation in Group Decisions, Studies in Informatics and Control, 12(2): 137-148, 2003.

[28] R.J. Harder, J.M. Keeter, B.W. Woodcock, J.W. Ferguson, F.W. Wills. Insights in Implementing Collaboration Engineering, Proceedings of the $38^{\text {th }}$ Hawaiian Internal Conference on System Sciences, IEEE Computer Society Press, 2005.

[29] M. de Hengst, E. de Kar, J. Appelman. Designing Mobile Information Services: User Requirements Elicitation with GSS Design and Application of a Repeatable Process, Proceedings of the $37^{\text {th }}$ Hawaiian Internal Conference on System Sciences, IEEE Computer Society Press, 2004.

[30] J.H. Appelman, J. Driel. Crisis-response in the Port of Rotterdam: can we do without a facilitator in distributed settings? Proceedings of the $38^{\text {th }}$ Hawaiian Internal Conference on System Sciences, IEEE Computer Society Press, 2005.

[31] B. Enserink. Creating a Scenariologic - Design and Application of a Repeatable Methodology, Proceedings of the $36^{\text {th }}$ Hawaiian Internal Conference on System Sciences, IEEE Computer Society Press, 2003.

[32] G.J. de Vreede, R.O. Briggs. Collaboration Engineering: Designing Repeatable Processes for HighValue Collaborative Tasks. Proceedings of the $38^{\text {th }}$ Hawaiian Internal Conference on System Sciences, IEEE Computer Society Press, 2005.

[33] L.R. Christensen. The Logic of Practices of Stigmergy: Representational Artifacts in Architectural Design, Proceedings of the 2008 ACM Conference on Computer Supported Cooperative Work, ACM, New York, 559-568, 2008.

[34] G.J. de Vreede, R.O. Briggs, G.L. Kolfschoten. ThinkLets: A Pattern Language for Facilitated and Practitioner-Guided Collaboration Processes, International Journal of Computer Applications in Technology, 25: 140-154, 2006.

[35] R.O. Briggs, B.A. Reinig, G.J. de Vreede. Meeting Satisfaction for technology-Supported Groups: An Empirical Validation of a Goal-Attainment Model, Small Group Research, 37, 2006.

[36] D. Luce. Individual Choice Behaviour, Wesley, New York., 1959.

[37] K.M. Carley. On Generating Hypotheses Using Computer Simulations. Proceedings of the 1999 International Symposium on Command and Control Research and Technology. Evidence Based Research, Vienna, 1999. 
[38] U. Wilensky. NetLogo, http://ccl.northwestern.edu/netlogo/, Center for Connected Learning and Computer-Based Modeling, Northwestern University, Evanston, 1999.

[39] C.B. Zamfirescu. Anthropocentric Group Decision Support Systems. PhD Thesis, Politehnica University of Bucharest, 2006.

[40] S. Guerin, D. Kunkle. Emergence of constraint in self-organizing systems. Nonlinear Dynamics, Psychology, and Life Sciences, 8(2): 131-146, 2004.

[41] B.E. Barbat, S.C. Negulescu, C.B. Zamfirescu. Human-Driven Stigmergic Control. Moving the Threshold. Proceedings of the $17^{\text {th }}$ IMACS World Congress - Scientific Computation, Applied Mathematics and Simulation, Paris, 2005.

[42] V.H.D. Parunak, S.A. Brueckner, J.A. Sauter, R. Matthews. Global convergence of local agent Behaviors, Proceedings of the $4^{\text {th }}$ International Joint Conferenece on Autonomouse Agents and Multi.Agent Systems (AAMAS), Utrecht, Netherlands, 2005.

[43] V.H.D. Parunak, S. Brueckner. Entropy and Self-Organization inMulti-Agent Systems. Proceedings of the Fifth International Conference on Autonomous Agents, Montreal, Canada, 124-130, 2001.

[44] B.E. Bărbat, C.B. Zamfirescu, S.C. Negulescu. The Best from Ants and Humans: Synergy in AgentBased Systems. Studies in Informatics and Control Journal, 13(1): 47-59, 2004.

[45] F.G. Filip, K. Leiviskä. Large-Scale Complex Systems. Handbook of Automation ( S. Nof, editor), 619-638, Springer, 2009.

Constantin-Bala Zamfirescu is currently associate professor (lecturer) at "Lucian Blaga" University of Sibiu, Romania. He took the PhD from "Politehnica" University Bucharest in Automation and Control field. His research interests include the employment of agent-oriented engineering paradigm in group decision support and manufacturing control systems. $\mathrm{He}$ is a member of IFAC Technical Committees 5.4, EU COST Action 298 and $2^{\text {nd }}$ EU NoE for the Advancement of Artificial Cognitive Systems, Interaction and Robotics.

Florin Gheorghe Filip member of the Romanian Academy (elected in 1991), received his M. $\mathrm{Sc}$ and Ph. D. in Control Engineering from the Technical University "Politehnica" Bucharest in 1970 and 1982, respectively. He has been with the National R \& D Institute for Informatics since 1970 and was the managing director of ICI from 1991 to 1997. He was elected in 2000 (and re-elected in 2002 and 2006) as a vice-president of the Romanian Academy. He has been the chair of the IFAC Technical Committees 5.4 (2002-2008). His main scienti?c interests include: control and optimization of large-scale systems, decision support systems, technology management, and models for knowledge-information society. He is author/co-author of six books, editor of nineteen volumes and the author/co-author of over two hundred articles published in contributed volumes and scientific journals. 